\title{
Cationic Graft Polymerization from Ultrafine Silica Initiated by Acylium Perchlorate Groups Introduced onto the Surface
}

\author{
Norio TsuboKawa and Akira Kogure \\ Department of Material and Chemical Engineering, Faculty of Engineering, \\ Niigata University, 8050, Ikarashi 2-nocho, Niigata 950-21, Japan
}

(Received August 4, 1992)

\begin{abstract}
The introduction of acylium perchlorate groups onto ultrafine silica was achieved by the reaction of silver perchlorate with acyl chloride groups, which were introduced by the treatment of carboxyl groups with thionyl chloride. The introduction of carboxyl groups onto silica surface was achieved by the hydrolysis of acid anhydride groups which introduced by the treatment of silica with 4-trimethoxysilyl tetrahydrophthalic anhydride. Surface acylium perchlorate groups introudced onto silica were capable of initiating cationic polymerization of styrene and polystyrene was effectively grafted onto the surface. The percentage of grafting increased to $65.4 \%$. The grafting efficiency was high during the first few conversion but rapidly decreased with an increase in conversion. This suggests that the grafted polymer propagates from acylium perchlorate groups and that ungrafted polymer is gradually formed by a chain transfer of growing polymer cation to the monomer. The cationic ring-opening polymerizations of tetrahydrofuran, styrene oxide, $\beta$-propiolactone, and $\varepsilon$-caprolactone were also initiated by acylium perchlorate groups on silica surface to give the corresponding polymer-grafted silica. The polymer-grafted silica gave a stable colloidal dispersion in good solvents for grafted polymer.
\end{abstract}

KEY WORDS Ultrafine Silica / Surface Grafting / Graft Polymerization / Ring-Opening Polymerization / Cyclic Ether / Lactone / Acylium Perchlorate Group / Dispersibility / 4-Trimethoxysilyltetrahydrophthalic Anhydride /

The dispersibility of inorganic ultrafine particles, such as silica, titanium oxide, and ferrite, in solvents and polymer matrices is remarkably improved by grafting of polymers onto these surfaces, because the grafted polymer chains on the surface interfere with the aggregation of ultrafine particles and increase the affinity of the surface for solvent. ${ }^{1}$

To prepare polymer-grafted ultrafine particles with a higher percentage of grafting, it is desirable to initiate the graft polymerization from initiating groups introduced onto these surfaces. We succeeded in the radical graft polymerization of vinyl monomers initiated by surface azo, ${ }^{2}$ peroxide, ${ }^{3}$ or peroxyester groups ${ }^{4}$ introduced onto ultrafine silica. In the polymerization, grafting was based on propagation from surface radicals formed by the decomposition of these initiating groups. We also reported that potassium carboxylate groups introduced onto ultrafine particles have ability to initiate the anionic ring-opening alternating polymerization of epoxides with cyclic acid anhydrides and the corresponding polymer, i.e., polyester, was grafted onto these surfaces. ${ }^{5}$ Furthermore, lithium silanolate groups introduced onto silica surface were capable of initiating anionic graft polymerization of methyl methacrylate and cyclic siloxanes. ${ }^{6}$

Cationic graft polymerization of polymers onto ultrafine silica has never been reported. In previous studies, we reported that acylium perchlorate groups introduced onto carbon black surface have ability to initiate the cationic polymerization of vinyl monomers ${ }^{7}$ 
and cationic ring-opening polymerization of lactones, ${ }^{8}$ cyclic ethers, ${ }^{9,10}$ and cyclic acetals $^{11}$ to give the corresponding polymergrafted carbon black.

In this article, the introduction of acylium perchlorate groups onto ultrafine silica sur- face (eq 1) and the cationic graft polymerization of several monomers from the surface acylium perchlorate groups (eq 2) are investigated. The stability of polymer-grafted silica dispersion in organic solvents is examined.
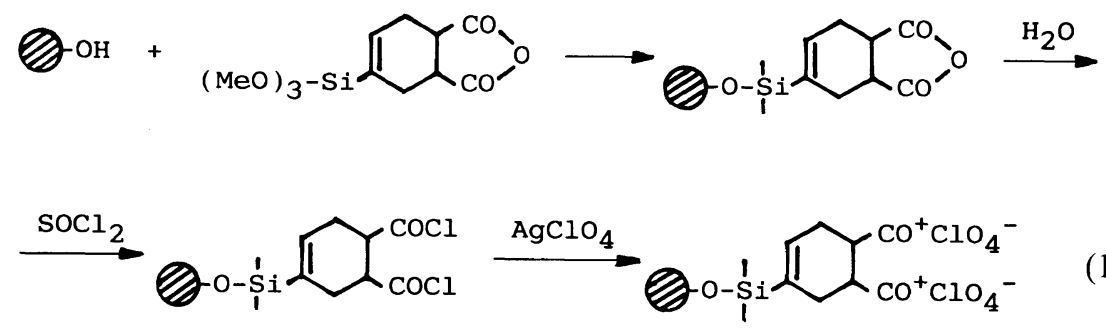

1

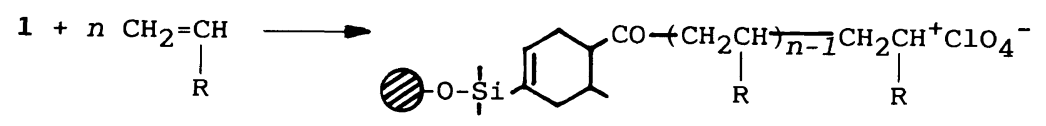

\section{EXPERIMENTAL}

\section{Materials}

Ultrafine silica used was Aerosil 200 (Nippon Aerosil Co., Japan). The BET specific surface area, particle size, and silanol group content were $200 \mathrm{~m}^{2} \mathrm{~g}^{-1}, 16 \mathrm{~nm}$, and $1.37 \mathrm{mmol} \mathrm{g}^{-1}$, respectively. Silanol group content was determined by measuring volumetrically the amount of ethane eveolved by the reaction with triethylaluminum. ${ }^{12,13}$ The silica was drid in vacuo at $110^{\circ} \mathrm{C}$ before use.

4-Trimethoxysilyltetrahydrophthalic anhydride (TSPA) obtained from Nissan Chemical Ind. Ltd., Japan, was used without further purification. Nitrobenzene was washed with dilute $\mathrm{H}_{2} \mathrm{SO}_{4}$, dried over calcium chloride, and distilled under reduced pressure. Toluene and benzene were washed with concentrated $\mathrm{H}_{2} \mathrm{SO}_{4}$, refluxed over sodium, and distilled.

Styrene was washed with aqueous alkali, dried over barium oxide, and distilled twice under reduced pressure. Tetrahydrofuran (THF) was refluxed over sodium, dried over sodium hydride, and distilled before used.
Epichlorohydrin (ECH), styrene oxide (SO), $\beta$-propiolactone (PL), and $\varepsilon$-caprolactone (CL) were dried over calcium hydride and distilled twice before use.

Guaranteed reagent grade silver perchlorate (Kojima Chemical Co., Ltd., Japan) was dried in vacuo at $120^{\circ} \mathrm{C}$ before use. Thionyl chloride was used without further purification. All other solvents and reagents were purified by general methods.

\section{Introduction of Carboxyl Groups onto Ultrafine \\ Silica}

The introduction of carboxyl groups onto silica surface was achieved by the hydrolysis of acid anhydride groups with water. The introduction of acid anhydride groups onto the silica surface was achieved by the reaction of silanol groups with TSPA in toluene. The detailed procedures are described in the previous paper. ${ }^{5}$

To hydrolyze surface acid anhydride groups, $5.0 \mathrm{~g}$ of silica was treated with $50 \mathrm{~cm}^{3}$ of water at room temperature for $1 \mathrm{~h}$. After treatment, the resulting silica was dried in vacuo at $50^{\circ} \mathrm{C}$. 
Introduction of Acyl Chloride Groups onto Ultrafine Silica

The introduction of acyl chloride groups onto silica surface was achieved by the treatment of surface carboxyl groups with thionyl chloride. A typical example is as follows. Into a flask equipped with a reflux condenser, $5.0 \mathrm{~g}$ of silica having carboxyl groups, 100 $\mathrm{cm}^{3}$ of benzene, and $30 \mathrm{~cm}^{3}$ of thionyl chloride were charged. The mixture was refluxed under stirring with a magnetic stirrer for $50 \mathrm{~h}$. After this treatment, benzene and unreacted thionyl chloride were removed under reduced pressure. The resulting silica was dried in vacuo at $90^{\circ} \mathrm{C}$ and stored in vacuo at room temperature.

\section{Introudction of Acylium Perchlorate Groups onto Ultrafine Silica}

The introduction of acylium perchlorate groups onto silica surface, i.e., pretreatment, was achieved by the reaction of acyl chloride groups with silver perchlorate in nitrobenzene. Pretreatment was required for the introduction of acylium perchlorate groups, because silver perchlorate is hardly soluble in nitrobenzene. ${ }^{7}$ A typical example is as follows. Into a $100 \mathrm{~cm}^{3}$ flask, $0.30 \mathrm{~g}$ of silica having acyl chloride groups, $0.20 \mathrm{~g}$ of silver perchlorate, and $5.0 \mathrm{~cm}^{3}$ of nitrobenzene were charged and the reaction mixture was stirred with a magnetic stirrer under dry nitrogen at room temperature for $48 \mathrm{~h}$.

\section{Graft Polymerization}

After the above pretreatment, $10.0 \mathrm{~cm}^{3}$ of monomer (styrene, PL, and CL) was added to the mixture and polymerization was conducted with stirring at $40^{\circ} \mathrm{C}$. After a prescribed polymerization time, the contents of the flask were poured into an excess of methanol to precipitate ungrafted polymer and polymer-grafted silica.

When THF and SO were used as monomers, polymerization was carried out without pretreatment, because silver perchlorate is sol- uble in these monomers. ${ }^{9}$ A typical example is as follows. Into a $100 \mathrm{~cm}^{3}$ flask, $0.30 \mathrm{~g}$ of silica having acyl chloride groups, $0.20 \mathrm{~g}$ of silver perchlorate were charged. The polymerization was initiated by the addition of $10.0 \mathrm{~cm}^{3}$ of monomer. In the polymerization of $\mathrm{SO}, 10.0 \mathrm{~cm}^{3}$ of carbon tetrachloride were used as solvent. After the reaction, the polymerization was stopped by the addition of methanol, and the unreacted monomer and solvent were removed by distillation under reduced pressure, yielding ungrafted polymer and polymer-grafted silica. ${ }^{9}$

The conversion was calculated by the following equation:

$$
\text { Conversion }(\%)=\frac{A}{B} \times 100
$$

$A$, Precipitate obtained (g)-Silica charged (g)

$B$, Monomer charged (g)

\section{Percentage of Grafting}

To separate the polymer-grafted silica from the reaction mixture containing ungrafted polymer, the product was dispersed in THF and the dispersion was centrifuged at $1.0 \times 10^{4}$ rpm until the particle precipitated completely. The silica precipitated was dispersed again in THF and centrifuged. This procedure was repeated until no polymer was detected in the supernatant solution. The percentage of grafting was calculated by the following equation:

$$
\text { Grafting }(\%)=\frac{\text { Polymer grafted }(\mathrm{g})}{\text { Silica charged }(\mathrm{g})} \times 100
$$

\section{Dispersibility of Polymer-Grafted Silica}

The stability of polymer-grafted silica dispersion in solvents was estimated as follows. ${ }^{5}$ Polymer-grafted silica $(0.30 \mathrm{~g})$ was dispersed in $100 \mathrm{~cm}^{3}$ of THF with a magnetic stirrer, and the dispersion was allowed to stand at room temperature. After a given time, $5.0 \mathrm{~cm}^{3}$ dispersion liquid were removed with 
a pipet, and the content of dispersed silica was determined. The stability of dispersion was determined by the following equation.

$$
\text { Silica dispersed }(\%)=\frac{A}{B} \times 100
$$

$$
\begin{aligned}
& A \text {, Silica dispersed after standing (g) } \\
& B \text {, Silica dispersed before standing }(\mathrm{g})
\end{aligned}
$$

\section{RESULTS AND DISCUSSION}

\section{Initiating Ability of Acylium Perchlorate Groups} on Silica

It is well known that the cationic polymerization of styrene and the ring-opening polymerization of THF are initiated by benzoyl or acetyl perchlorate prepared by the reaction of acid chloride with silver perchlorate. ${ }^{14,15}$ We reported the cationic grafting of polystyrene, ${ }^{7}$ polyesters,${ }^{8}$ polyethers,,${ }^{9,10}$ and polyacetal $^{11}$ from carbon black surface by use of carbon black having acylium perchlorate groups as initiator. The polymerization of styrene was carried out under several conditions using silica having acyl chloride groups (Silica-COCl) and silver perchlorate. The results are summarized in Table I. As shown in Table I, styrene was not polymerized by silver perchlorate, untreated silica, or Silica$\mathrm{COCl}$ alone (Run nos. 1, 2, and 4). In addition, polymerization could not be detected even if

Table I. Polymerization of styrene under several conditions ${ }^{\mathrm{a}}$

\begin{tabular}{clcccc}
\hline \multirow{2}{*}{$\begin{array}{c}\text { Run } \\
\text { no. }\end{array}$} & Silica & $\mathrm{AgClO}_{4}$ & & Conversion & Grafting \\
\cline { 5 - 5 } & & $\mathrm{g}$ & & $\%$ & $\%$ \\
\hline 1 & None & 0.20 & & 0 & - \\
2 & Untreated & - & & 0 & - \\
3 & Untreated & 0.20 & & 0 & - \\
4 & Silica-COCl & - & & 0 & - \\
5 & Silica-COCl & 0.20 & & 46.5 & 64.5
\end{tabular}

${ }^{a}$ Pretreatment: Silica, $0.30 \mathrm{~g} ; \mathrm{C}_{6} \mathrm{H}_{5} \mathrm{NO}_{2}, \quad 5.0 \mathrm{~cm}^{3}$; room temp, $48 \mathrm{~h}$. Polymerization: styrene, $10.0 \mathrm{~cm}^{3}$; $40^{\circ} \mathrm{C} ; 10 \mathrm{~h}$. untreated silica was pretreated with silver perchlorate (Run no. 3).

The polymerization of styrene was initiated by Silica- $\mathrm{COCl}$ pretreated with silver perchlorate (Run no. 5) to give polystyrene grafted silica. The results indicate that acylium perchlorate groups formed by the reaction of acyl chloride groups with silver perchlorate initiate the polymerization and grafted polymer chains propagate from silica surface.

The acylium perchlorate group content of silica after pretreatment for $48 \mathrm{~h}$ at room temperature in nitrobenzene was estimated to be $0.35 \mathrm{mmol} \mathrm{g}^{-1}$ by titration. ${ }^{16}$ The amount of acid anhydride groups introduced onto silica by treatment with TSPA was $0.96 \mathrm{mmol}$ $\mathrm{g}^{-1}$.5) This indicates that about $18 \%$ of acid anhydride groups are converted to acylium perchlorate groups, because one acid anhydride group can generate two acylium perchlorate groups.

Figure 1 shows the time-conversion curve of the styrene polymerization initiated by acylium perchlorate groups introduced onto silica. The conversion linearly increased with polymerization time and reached $46.5 \%$ after $10 \mathrm{~h}$.

\section{Cationic Grafting of Polystyrene}

Figure 2 shows the relationship between

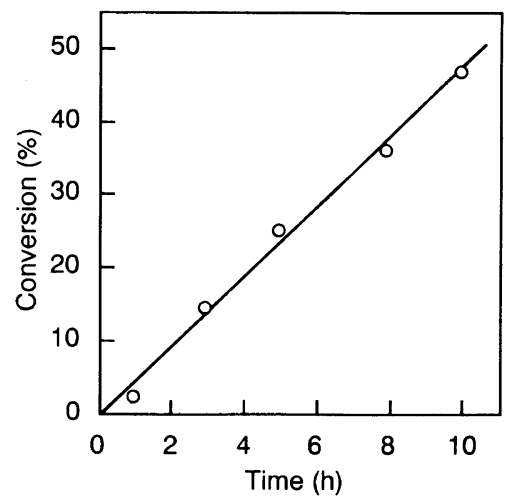

Figure 1. Cationic polymerization styrene initiated by acylium perchlorate groups introduced onto silica surface. Pretreatment: Silica-COCl, $0.30 \mathrm{~g} ; \mathrm{AgClO}_{4}, 0.20 \mathrm{~g}$; $\mathrm{C}_{6} \mathrm{H}_{5} \mathrm{NO}_{2}, 5.0 \mathrm{~cm}^{3}$; room temp, $48 \mathrm{~h}$. Polymerization: styrene, $10.0 \mathrm{~cm}^{3}, 40^{\circ} \mathrm{C}$. 
conversion and percentage of grafting (or grafting efficiency) for the graft polymerization of styrene shown in Figure 1; (Grafting efficiency in $\%)=100 \times($ weight of grafted polymer)/(total weight of both grafted and ungrafted polymers). As shown in Figure 2, the percentage of grafting increased gradually with conversion and reached $64.5 \%$ after $10 \mathrm{~h}$.
Grafting efficiency was high during the first few conversion but rapidly decreased with increasing conversion. This suggests that the grafted polymer propagates from acylium perchlorate groups and that the ungrafted polymer is gradually formed by a chain transfer of growing polymer cation to the monomer (eq 3).

$$
{ }_{m \mathrm{CH}_{2}=\left.\right|_{\mathrm{R}} \longrightarrow \mathrm{C}_{\mathrm{R}}}^{\longrightarrow}
$$

Figure 3 shows the infrared spectra of untreated and polystyrene-grafted silica. As shown in Figure 3, the infrared spectra of polystyrene-grafted silica exhibited adsorptions at 1605,1498 , and $1454 \mathrm{~cm}^{-1}$, which characteristic of polystyrene, and at 1780

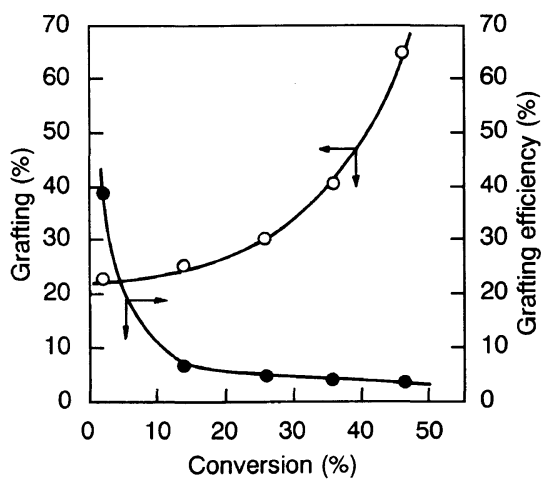

Figure 2. Relationship between conversion and percentage of grafting (grafting efficiency) of polystyrene onto silica. Polymerization conditions are given in Figure 1. $\mathrm{cm}^{-1}$, which characteristic of carbonyl group arising from TSPA moieties on silica surface. The results clearly show the grafting of polystyrene onto the silica surface.

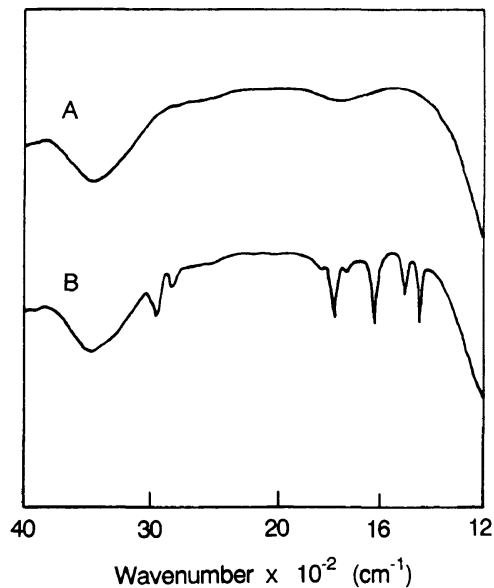

Figure 3. Infrared spectra $(\mathrm{KBr})$ of $(\mathrm{A})$ untreated silica and (B) polystyrene-grafted silica. 
Table II. Cationic ring-opening polymerization of cyclic ethers and lactones initiated by acylium perchlorate groups on silica surface

\begin{tabular}{|c|c|c|c|c|}
\hline \multirow{2}{*}{ Monomer } & \multirow{2}{*}{ Promoter $^{\mathrm{a}}$} & Time & Conversion & Grafting \\
\hline & & $\mathrm{h}$ & $\%$ & $\%$ \\
\hline $\mathrm{THF}$ & - & 52 & $0.2^{\mathrm{b}}$ & 19.5 \\
\hline THF & $\mathrm{ECH}$ & 52 & $5.4^{\mathrm{b}}$ & 44.2 \\
\hline $\mathrm{SO}$ & - & 2 & $8.8^{\mathrm{b}, \mathrm{c}}$ & 27.7 \\
\hline PL & - & 10 & $31.5^{\mathrm{d}}$ & 19.6 \\
\hline $\mathrm{CL}$ & - & 10 & $70.9^{d}$ & 18.7 \\
\hline
\end{tabular}

a $1.0 \mathrm{~mol} \%$.

b Silica, $0.30 \mathrm{~g} ; \mathrm{AgClO}_{4}, 0.20 \mathrm{~g}$; monomer, $10.0 \mathrm{~cm}^{3}$; $40^{\circ} \mathrm{C}$.

c Carbon tetrachloride $\left(10.0 \mathrm{~cm}^{3}\right)$ was used as solvent.

d Polymerization conditions are given in Figure 1.

Ring-Opening Polymerization Initiated by Acylium Perchlorate Groups on Silica

The cationic grafting of polyethers and polyester onto silica surface by the ringopening polymerization of cyclic ethers, such as THF and SO, and lactones, such as PL and CL, initiated by acylium perchlorate groups was examined. The results are summarized in Table II.

In the absence of promoter, the ring-opening polymerization rate of THF initiated by acylium perchlorate groups on silica was very small. In the presence of $\mathrm{ECH}$ as promoter, the polymerization of THF was initiated by acylium perchlorate groups to give poly(THF) grafted silica. It was found that acylium perchlorate groups introduced onto silica surface have an ability to initiate the cationic ring-opening polymerization of $\mathrm{SO}$ and poly(SO) was grafted from the surface. The polymerization rate of $\mathrm{SO}$ was very large so that carbon tetrachloride was added as solvent.

Acylium perchlorate groups on silica surface were capable of initiating the cationic ring-opening polymerization of lactones, such as PL and CL, to give poly(PL)- and Poly(CL)grafted silica, respectively.

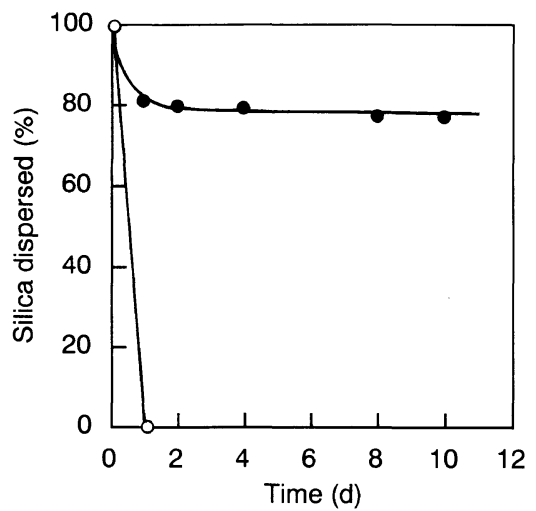

Figure 4. Stability of polystyrene-grafted silica dispersion in THF at room temperature. (O), untreated silica; (O), polystyrene-grafted silica (grafting $=64.5 \%$ ).

\section{Stability of Polystyrene-Grafted Silica Disper-} sion

The stability of polystyrene-grafted silica dispersion in THF was compared with that of untreated one. The rsults are shown in Figure 4. As shown in Figure 4, untreated silica precipitated completely within 1 day. Polystyrene-grafted silica gave a stable colloidal dispersion in THF. This indicates that the grafted polystyrene chain on the surface interfere with the aggregation of silica particles.

Estimation of particle size distribution of polymer-grafted silica by dynamic light scattering method is now under investigation.

\section{CONCLUSIONS}

1. Acylium perchlorate groups were successfully introduced onto silica surface by the reaction of surface acyl chloride groups with silver perchlorate.

2. The cationic polymerization of styrene and ring-opening polymerization of cyclic ethers and lactones were initiated by acylium perchlorate groups introduced onto silica surface.

3. In the polymerizations, the corresponding polymers were grafted onto silica surface based on the propagation of the polymers 
from the surface.

4. Polymer-grafted silica gave a stable colloidal dispersion in good solvents for grafted polymer chain.

\section{Acknowledgments. We thank Nissan Chem-} ical Ind. Ltd., Japan for providing 4-trimethoxysilyltetrahydrophthalic anhydride.

\section{REFERENCES}

1. N. Tsubokawa, Prog. Polym. Sci., in press.

2. N. Tsubokawa, A. Kogure, K. Maruyama, Y. Sone, and M. Shimomura, Polym. J., 22, 827 (1990).

3. N. Tsubokawa and H. Ishida, J. Polym. Sci., A: Polym. Chem., 30, 224 (1992).

4. N. Tsubokawa and H. Ishida, Polym. J., 24, 809 (1992).

5. N. Tsubokawa, A. Kogure, and Y. Sone, J. Polym. Sci., A: Polym. Chem., 28, 1923 (1990).
6. N. Tsubokawa and T. Umeno, unpublished results.

7. N. Tsubokawa, J. Polym. Sci., Polym. Chem. Ed., 22, 1515 (1984).

8. N. Tsubokawa, J. Appl. Polym. Sci., 30, 2041 (1985).

9. N. Tsubokawa, H. Nunokawa, and Y. Sone, $J$. Macromol. Sci.-Chem., A23, 105 (1986).

10. N. Tsubokawa, Yu Jian, and Y. Sone, J. Polym. Sci., Polym. Chem. Ed., 26, 2715 (1988).

11. N. Tsubokawa, J. Polym. Sci., Polym. Chem. Ed., 25, 1979 (1987).

12. M. Sato, Y. Kanbayashi, K. Kobayashi, and Y. Sima, J. Catalysis, 1, 342 (1976).

13. S. Matsuda and S. Okazaki, Nippon Kagaku Kaishi, 1287 (1986).

14. W. R. Longworth and P. H. Plesch, Proc. Chem. Soc., 117 (1958).

15. P. Dreyfuss and J. P. Kennedy, J. Polym. Sci., Polym. Lett. Ed., 14, 139 (1976).

16. N. Tsubokawa, Yu Jian, and Y. Sone, Polym. Bull., 16, 249 (1986). 